\title{
Effect of PSCA gene polymorphisms on gastric cancer risk and survival prediction: A meta-analysis
}

\author{
TAO ZHANG ${ }^{1}$, YUAN-NENG CHEN $^{1}$, ZHEN WANG $^{2}$, JUN-QIANG CHEN $^{2}$ and SHI HUANG ${ }^{1}$ \\ ${ }^{1}$ Department of Gastroenterology, Ruikang Hospital of Guangxi Traditional Chinese Medical University, Nanning 530011; \\ ${ }^{2}$ Department of Gastrointestinal Surgery, The First Affiliated Hospital of Guangxi Medical University, \\ Nanning 530021, Guangxi Zhuang Autonomous Region, P.R. China
}

Received February 24, 2012; Accepted April 13, 2012

DOI: $10.3892 /$ etm.2012.563

\begin{abstract}
Previous studies have shown that two single-nucleotide polymorphisms (SNPs) in PSCA (rs2976392 and rs2294008) are associated with gastric cancer (GC), but the results are conflicting. Additionally, the prognostic value of PSCA gene polymorphisms for GC patients is unknown. We performed a meta-analysis using 9 eligible case-control studies to investigate the association between PSCA polymorphisms and GC risk, and additionally investigated the prognostic value of PSCA polymorphisms for GC patients with two eligible studies. The association was measured using random-effect or fixed-effect odds ratios (ORs) combined with 95\% confidence intervals (CIs) according to the heterogeneity of the studies. We found that rs2294008 (dominant model: OR, 1.44; 95\% CI, 1.16-1.79) and rs2976392 (dominant model: OR, 1.41; 95\% CI, 0.98-2.04) polymorphisms were associated with increased risk of GC, although the association of rs2976392 was not statistically significant. For rs2294008, the associations were all consistently significant among the different subgroups stratified by ethnicity and tumor location, but not significant in intestinal or diffuse subtypes. For rs2976392, the associations were consistently significant for the intestinal, diffuse and non-cardia subtypes, but not significant for the cardia subtype. Furthermore, two eligible studies reported inverse results of PCSA in predicting the survival of GC patients (HR, 0.75; 95\% CI, 0.59-0.96; and HR, 2.12; 95\% CI, 1.22-3.69, respectively). In conclusion, PSCA gene
\end{abstract}

Correspondence to: Professor Yuan-Neng Chen, Department of Gastroenterology, Ruikang Hospital of Guangxi Traditional Chinese Medical University, 10 Huadong Road, Nanning 530011, Guangxi Zhuang Autonomous Region, P.R. China

E-mail: cyn60668@yahoo.com.cn

Dr Zhen Wang, Department of Gastrointestinal Surgery, The First Affiliated Hospital of Guangxi Medical University, 6 Shuangyong Road, Nanning 530021, Guangxi Zhuang Autonomous Region, P.R. China

E-mail: wangzhensurgeon@163.com

Key words: PSCA, gastric cancer, meta-analysis polymorphisms are associated with increased risk of $\mathrm{GC}$ and are correlated with the prognosis of GC patients. Future studies are required to evaluate the molecular mechanisms of PSCA polymorphisms in GC and validate the prognostic value in a larger number of patients.

\section{Introduction}

Gastric cancer (GC) remains a major public health issue worldwide, with the highest incidence in China, Japan and Eastern European countries, despite a steady decrease in incidence and mortality in the past 30 years $(1,2)$. The etiology of GC has a clear environmental component characteristic of the geographically varied incidence in its distribution $(1,2)$. Over the years, several environmental factors, including diet, tobacco smoking and Helicobacter pylori infection, have been shown to be responsible for gastric carcinogenesis $(2,3)$. Besides environmental factors, genetic factors also play an important role in the development of GC, and this is confirmed by the fact that only a small proportion of individuals exposed to known environmental risk factors develop GC (4,5). Additionally, recent studies have shown that single-nucleotide polymorphisms (SNPs) in genes encoding tumor necrosis factor $\alpha, \mathrm{COX}-2$, and CD14 are associated with increased GC risk (5-7), indicating that genetic variation contributes to GC carcinogenesis. However, the molecular mechanism of GC pathogenesis is still unknown.

Located on chromosome 8q24.2, the prostate stem cell antigen (PSCA) gene encodes a 123-amino acid glycoprotein, which is a cell surface antigen. PSCA was first identified as a prostate-specific antigen overexpressed in prostate cancers, including metastatic and hormone-refractory cancers. However, it is also expressed in other solid tumors, including ovarian mucinous and pancreatic cancer, renal-cell carcinoma and bladder cancer $(8,9)$. In contrast with observations in other tumors, PSCA expression is downregulated in GC (10). Although an in vitro cell-proliferation inhibitory activity of PSCA was reported, the regulation of PSCA expression and its physiological function are still largely unknown $(9,11)$. Previously, genome-wide association studies (GWAS) have found that two SNPs in PSCA, rs2976392 and rs2294008, are significantly associated with GC in Chinese, Japanese, Korean and Caucasian individuals, particularly for diffuse-type GC 
(11-13). However, the sample size of these studies was small, and the association between SNPs (rs2976392 and rs2294008) in the PSCA gene and susceptibility to intestinal-type GC remains controversial $(11,13)$. In addition, the prognostic value of PSCA gene polymorphisms for GC patients requires additional study.

The aim of this study was to perform a systematic review and meta-analysis concerning: i) the association between PSCA gene polymorphisms and GC risk; ii) the influence of this polymorphism on survival outcomes in GC patients.

\section{Materials and methods}

Search strategy. The literature was searched using PubMed and EMBASE to identify relevant and available published articles. The upper limit of the search date was not specified and the lower limit was July 2011. The following search terms were used: gastric cancer/carcinoma/tumor/neoplasm, stomach cancer/carcinoma/tumor/neoplasm, prostate stem cell antigen/PSCA, and polymorphism (rs2976392 or rs2294008). Free text and MeSH search for keywords were employed. The language in which the papers were written was not restricted. To search for more potentially relevant trials, reference lists from studies included in the electronic search were screened.

Inclusion and exclusion criteria. The inclusion criteria of this meta-analysis were as follows: i) independent case-control or cohort studies (for humans only); and ii) studies providing complete case and control data regarding the association between PSCA gene polymorphism (rs2976392 or rs2294008) and GC risk (or survival outcomes of GC patients). The exclusion criteria were: i) no controls; ii) incomplete data; and iii) duplicate publications.

Data extraction. Information was carefully extracted from all eligible studies independently by two researchers (Zhen Wang and Jun-Qiang Chen) according to the above-mentioned inclusion criteria. The following variables were extracted if available: first author, publication time, country and ethnicity of the sample, genotyping method, sample size (numbers of GC patients and control subjects) and the results of studies. Where a study reported results on different sub-populations according to ethnicity, we considered each sub-population as a separate study in our meta-analysis. Final agreement was obtained through discussion.

Statistical analysis. The association of PSCA gene polymorphisms with GC risk and survival outcomes in GC patients were assessed by pooled odds (ORs) or hazard ratios (HRs) with their corresponding $95 \%$ confidence intervals (CIs), respectively. Statistical heterogeneity between and within groups were measured by using the $\mathrm{Q}$ test, and $\mathrm{P}<0.1$ was considered to indicate statistical significance. The fixed-effects model was used to pool the data, but the random-effects model was used if statistical heterogeneity $\left(\mathrm{P}_{\mathrm{h}}<0.1\right)$ was found. Additionally, subgroup analysis was conducted on the basis of ethnicity, Lauren's classification, and tumor location of GC in order to establish the effect of clinical heterogeneity. Funnel plots were used to assess publication bias. All of the calculations were

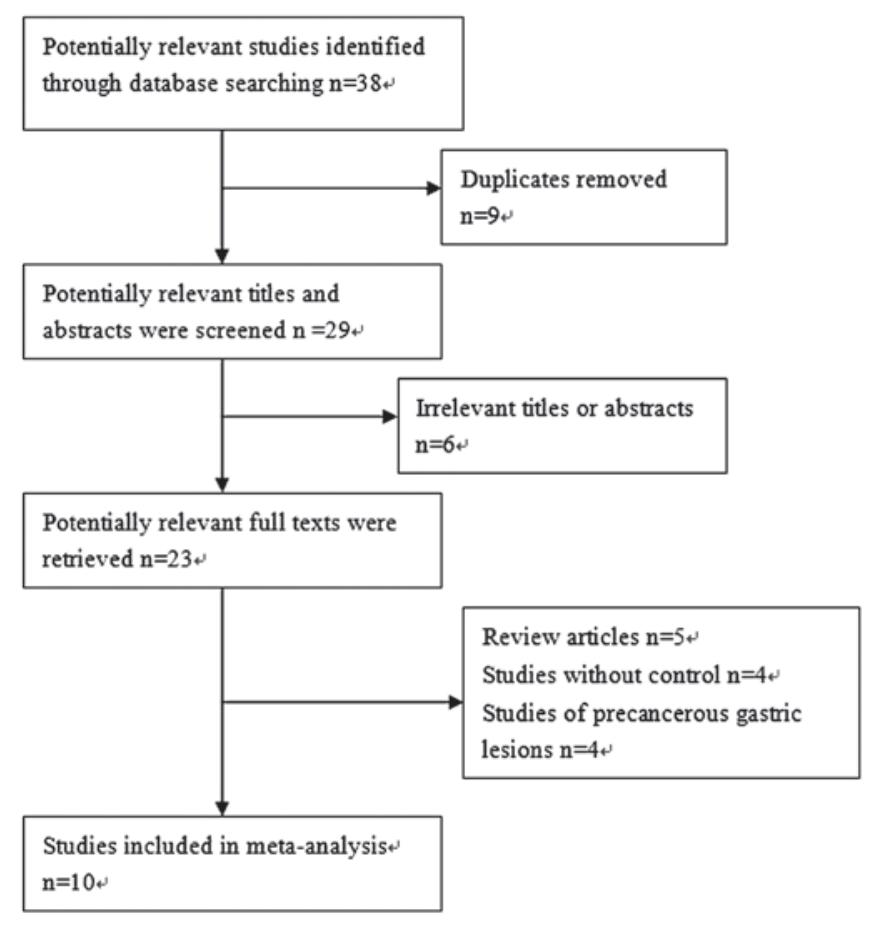

Figure 1. QUORUM flow chart for studies.

performed using Revman version 5.1 (14), and all statistical tests were two-sided.

\section{Results}

Study selection and description. According to the search strategy above, a total of 38 studies were yielded: 23 in Pubmed and 15 in Embase. Through the step of screening the titles and abstracts, a number of articles were found to be irrelevant or were identified in more than one database. Therefore, 23 articles remained for potential inclusion and were obtained in full-text version. Following review of the full text, 13 articles were excluded. The main reasons for excluding studies were as follows: i) review articles; ii) studies without control; and iii) studies of precancerous gastric lesions. A total of 10 studies (11-13,15-21) were eligible for this meta-analysis. The study selection process is shown in Fig 1. Among the 10 included studies, 9 studies analyzed the association between SNPs (9 studies of rs2294008 polymorphism and 5 studies of rs2976392 polymorphism) and GC risk (11-13,15-20); and 2 studies analyzed the association between rs2294008 polymorphism and the survival of GC patients $(20,21)$. Notably, the study by Wang et al (21) only analyzed the association between the rs2294008 polymorphism and the survival of GC patients, thus there was no control in this study.

Table I lists the main characteristics of the 10 included studies, including first author, published year, original country, ethnicity, genotyping method and number of cases and controls. Tables II and III list genotype distribution of rs2294008 and rs2976392 polymorphisms. The distribution of genotypes in the controls was consistent with Hardy-Weinberg equilibrium in all studies except one (which did not report clearly) (11). 
Table I. Characteristics of studies included in the meta-analysis.

\begin{tabular}{|c|c|c|c|c|c|c|c|}
\hline First author/(ref) & Year & Country & Ethnicity & $\begin{array}{c}\text { Genotyping } \\
\text { method }\end{array}$ & Cases & Controls & Genes \\
\hline Ou (15) & 2010 & China & Asian & PCR/LDR & 196 & 246 & $\begin{array}{c}\text { rs2294008 and } \\
\text { rs2976392 }\end{array}$ \\
\hline Song (16) & 2011 & Korea & Asian & PCR-RFLP & 3245 & 1700 & rs2294008 \\
\hline Lochhead a (12) & 2011 & Poland & Caucasian & PCR & 312 & 383 & rs2294008 \\
\hline Lochhead b (12) & 2011 & USA & Caucasian & PCR & 309 & 211 & rs2294008 \\
\hline Sakamoto a (11) & 2008 & Japan & Asian & PCR & 1531 & 1398 & $\begin{array}{l}\text { rs2294008 and } \\
\text { rs2976392 }\end{array}$ \\
\hline Sakamoto b (11) & 2008 & Korea & Asian & PCR & 875 & 390 & $\begin{array}{c}\text { rs2294008 and } \\
\text { rs2976392 }\end{array}$ \\
\hline $\mathrm{Lu}(13)$ & 2010 & China & Asian & PCR-RFLP & 1053 & 1100 & $\begin{array}{l}\text { rs2294008 and } \\
\text { rs2976392 }\end{array}$ \\
\hline Zeng (20) & 2011 & China & Asian & PCR-RFLP & 460 & 549 & rs2294008 \\
\hline $\mathrm{Wu}(17)$ & 2009 & China & Asian & PCR-RFLP & 1736 & 1020 & $\begin{array}{c}\text { rs2294008 and } \\
\text { rs2976392 }\end{array}$ \\
\hline Matsuo (18) & 2009 & Japan & Asian & PCR & 708 & 708 & $\begin{array}{l}\text { rs2294008 and } \\
\text { rs2976392 }\end{array}$ \\
\hline Sala (19) & 2011 & $\begin{array}{c}10 \text { European } \\
\text { countries }\end{array}$ & Caucasian & PCR & 411 & 1530 & rs2294008 \\
\hline Wang (21) & 2010 & China & Asian & PCR & 943 & no & rs2294008 \\
\hline
\end{tabular}

PCR, polymerase chain reaction; LDR, ligase detection reaction; RFLP, restriction fragment length polymorphism.

Table II. Genotype distribution of rs2294008.

\begin{tabular}{|c|c|c|c|c|c|c|c|c|c|c|c|}
\hline \multirow[t]{2}{*}{ Study/(ref) } & \multicolumn{5}{|c|}{ Cases } & \multicolumn{5}{|c|}{ Controls } & \multirow[t]{2}{*}{ HWE } \\
\hline & $\mathrm{CC}$ & CT & TT & $\mathrm{C}(\%)$ & $\mathrm{T}(\%)$ & $\mathrm{CC}$ & CT & TT & $\mathrm{C}(\%)$ & $\mathrm{T}(\%)$ & \\
\hline Ou (15) & 85 & 93 & 18 & $263(67.1)$ & $129(32.9)$ & 132 & 96 & 18 & $360(73.2)$ & $132(26.8)$ & $>0.05$ \\
\hline Song (16) & 576 & 1620 & 1049 & $2772(42.7)$ & $3718(57.3)$ & 414 & 818 & 468 & $1646(48.4)$ & 1754 (51.6) & 0.13 \\
\hline Lochhead a (12) & 47 & 143 & 102 & $237(40.6)$ & 347 (59.4) & 101 & 166 & 115 & $368(48.2)$ & $396(51.8)$ & $>0.05$ \\
\hline Lochhead b (12) & 85 & 129 & 94 & $299(48.5)$ & $317(51.5)$ & 49 & 110 & 49 & $208(50.0)$ & $208(50.0)$ & $>0.05$ \\
\hline Sakamoto a (11) & 96 & 700 & 728 & $892(29.3)$ & $2156(70.7)$ & 210 & 650 & 536 & $1070(38.3)$ & $1722(61.7)$ & NR \\
\hline Sakamoto b (11) & 133 & 461 & 277 & $727(41.7)$ & $1015(58.3)$ & 122 & 176 & 92 & $420(53.8)$ & $360(46.2)$ & NR \\
\hline $\mathrm{Lu}(13)$ & 547 & 404 & 72 & $1498(73.2)$ & $548(26.8)$ & 605 & 387 & 77 & 1597 (74.7) & $541(25.3)$ & 0.166 \\
\hline Zeng (20) & 202 & 216 & 42 & $620(67.4)$ & $300(32.6)$ & 289 & 223 & 37 & $801(73.0)$ & $297(27.0)$ & 0.493 \\
\hline Wu (17) & 759 & 819 & 132 & $2337(68.3)$ & 1083 (31.7) & 506 & 412 & 77 & $1424(71.6)$ & $566(28.4)$ & $>0.05$ \\
\hline Matsuo (18) & 330 & 329 & 49 & 989 (69.8) & $427(30.2)$ & 273 & 338 & 97 & $884(62.4)$ & $532(37.6)$ & 0.64 \\
\hline Sala (19) & 93 & 198 & 118 & $384(46.9)$ & $434(53.1)$ & 491 & 714 & 310 & $1696(56.0)$ & $1334(44.0)$ & $>0.05$ \\
\hline
\end{tabular}

HWE, Hardy-Weinberg equilibrium; NR, not reported.

Table IV lists the main results of the meta-analysis of PSCA gene (rs2976392 or rs2294008) polymorphism and GC risk.

rs2294008 polymorphism and GC risk. Nine studies were eligible for meta-analysis (11-13,15-20), and the results of the meta-analysis indicated that $\mathrm{rs} 2294008 \mathrm{C} / \mathrm{T}$ polymorphism of the PSCA gene was significantly associated with susceptibility to $\mathrm{GC}$ (dominant model: OR, 1.44; 95\% CI, 1.16-1.79; recessive model: OR, 1.20; 95\% CI, 1.01-1.42; additive model: OR, 1.23; 95\% CI, 1.08-1.40). Significant between-study heterogeneity was detected in all genetic models $\left(\mathrm{P}_{\mathrm{h}}<0.1\right)$. We therefore performed subgroup analysis according to ethnicity, Lauren's 
Table III. Genotype distribution of rs2976392.

\begin{tabular}{|c|c|c|c|c|c|c|c|c|c|c|c|}
\hline \multirow[t]{2}{*}{ Study/(ref) } & \multicolumn{5}{|c|}{ Cases } & \multicolumn{5}{|c|}{ Controls } & \multirow[t]{2}{*}{ HWE } \\
\hline & GG & GA & AA & $\mathrm{G}(\%)$ & $\mathrm{A}(\%)$ & GG & GA & AA & $\mathrm{G}(\%)$ & $\mathrm{A}(\%)$ & \\
\hline Ou (15) & 99 & 85 & 12 & $283(72.2)$ & 109 (27.8) & 130 & 102 & 14 & $362(73.6)$ & $130(26.4)$ & $>0.05$ \\
\hline Sakamoto a (11) & 97 & 691 & 737 & $885(29.0)$ & $2165(71.0)$ & 211 & 650 & 536 & $1072(38.4)$ & $1722(61.6)$ & NR \\
\hline Sakamoto b (11) & 134 & 453 & 278 & $721(41.7)$ & $1009(58.3)$ & 122 & 175 & 93 & $419(53.7)$ & $361(46.3)$ & NR \\
\hline $\mathrm{Lu}(13)$ & 500 & 464 & 79 & $1464(70.2)$ & $622(29.8)$ & 602 & 402 & 78 & $1606(74.2)$ & $558(25.8)$ & 0.336 \\
\hline $\mathrm{Wu}(17)$ & 789 & 793 & 142 & $2371(68.8)$ & 1077 (31.2) & 492 & 429 & 81 & $1413(70.5)$ & $591(29.5)$ & $>0.05$ \\
\hline Matsuo (18) & 331 & 328 & 48 & $990(70.0)$ & $424(30.0)$ & 274 & 337 & 96 & $885(62.6)$ & $529(37.4)$ & 0.64 \\
\hline
\end{tabular}

HWE, Hardy-Weinberg equilibrium; NR, not reported.

Table IV. Results of the meta-analysis (OR).

\begin{tabular}{|c|c|c|c|c|c|c|c|}
\hline Polymorphism & Overall & Asians & Caucasians & Diffuse & Intestinal & Cardia & Non-cardia \\
\hline \multicolumn{8}{|l|}{ rs2294008 C/T } \\
\hline CT/TT vs. CC (D) & $\begin{array}{c}1.44 \mathrm{r} \\
(1.16,1.79)\end{array}$ & $\begin{array}{c}1.46 \mathrm{r} \\
(1.13,1.90)\end{array}$ & $\begin{array}{c}1.37 \mathrm{r} \\
(0.87,2.16)\end{array}$ & $\begin{array}{c}1.42 \mathrm{r} \\
(0.89,2.27)\end{array}$ & $\begin{array}{c}1.30 \mathrm{r} \\
(0.91,1.86)\end{array}$ & $\begin{array}{c}1.21 \mathrm{f} \\
(1.03,1.42)\end{array}$ & $\begin{array}{c}1.45 \mathrm{f} \\
(1.26,1.66)\end{array}$ \\
\hline TT vs. CT/CC (R) & $\begin{array}{c}1.20 \mathrm{r} \\
(1.01,1.42)\end{array}$ & $\begin{array}{c}1.12 \mathrm{r} \\
(0.90,1.39)\end{array}$ & $\begin{array}{c}1.44 \mathrm{f} \\
(1.20,1.72)\end{array}$ & $\begin{array}{c}1.04 \mathrm{r} \\
(0.60,1.80)\end{array}$ & $\begin{array}{c}1.05 \mathrm{r} \\
(0.76,1.46)\end{array}$ & $\begin{array}{c}1.00 \mathrm{f} \\
(0.76,1.31)\end{array}$ & $\begin{array}{c}1.36 \mathrm{f} \\
(1.11,1.68)\end{array}$ \\
\hline T vs. C (A) & $\begin{array}{c}1.23 \mathrm{r} \\
(1.08,1.40)\end{array}$ & $\begin{array}{c}1.22 \mathrm{r} \\
(1.04,1.43)\end{array}$ & $\begin{array}{c}1.33 \mathrm{f} \\
(1.19,1.49)\end{array}$ & $\begin{array}{c}1.13 \mathrm{r} \\
(0.85,1.52)\end{array}$ & $\begin{array}{c}1.14 \mathrm{r} \\
(0.89,1.46)\end{array}$ & $\begin{array}{c}1.12 \mathrm{f} \\
(0.99,1.26)\end{array}$ & $\begin{array}{c}1.31 \mathrm{f} \\
(1.19,1.45)\end{array}$ \\
\hline \multicolumn{8}{|l|}{ rs2976392 A/G } \\
\hline GA/AA vs. GG (D) & $\begin{array}{c}1.41 \mathrm{r} \\
(0.98,2.04)\end{array}$ & - & - & $\begin{array}{c}2.80 \mathrm{r} \\
(1.43,5.47)\end{array}$ & $\begin{array}{c}1.46 \mathrm{f} \\
(1.27,1.69)\end{array}$ & $\begin{array}{c}1.03 \mathrm{f} \\
(0.85,1.25)\end{array}$ & $\begin{array}{c}1.20 \mathrm{f} \\
(1.01,1.43)\end{array}$ \\
\hline AA vs. GA/GG (R) & $\begin{array}{c}1.05 \mathrm{r} \\
(0.75,1.48)\end{array}$ & - & - & $\begin{array}{c}1.67 \mathrm{f} \\
(1.45,1.92)\end{array}$ & $\begin{array}{c}1.22 \mathrm{f} \\
(1.05,1.42)\end{array}$ & $\begin{array}{c}0.82 \mathrm{f} \\
(0.57,1.20)\end{array}$ & $\begin{array}{c}1.04 \mathrm{f} \\
(0.75,1.42)\end{array}$ \\
\hline A vs. G (A) & $\begin{array}{c}1.17 \mathrm{r} \\
(0.93,1.48)\end{array}$ & - & - & $\begin{array}{c}2.18 \mathrm{r} \\
(1.21,3.91)\end{array}$ & $\begin{array}{c}1.26 \mathrm{f} \\
(1.15,1.38)\end{array}$ & $\begin{array}{c}0.99 \mathrm{r} \\
(0.85,1.15)\end{array}$ & $\begin{array}{c}1.12 \mathrm{f} \\
(0.98,1.28)\end{array}$ \\
\hline
\end{tabular}

A, additive model; D, dominant model; $R$, recessive model; r, random-effects model $\left(\mathrm{P}_{\mathrm{h}}<0.1\right)$; f, fixed-effects model $\left(\mathrm{P}_{\mathrm{h}}>0.1\right)$.

classification, and tumor location. In the subgroup analysis based on ethnicity, significant association was detected in both Asians (dominant and additive model, Table IV) and Caucasians (recessive and additive model, Table IV). Notably, in subgroup analysis based on Lauren's classification, no significant association was detected for either diffuse-type or intestinal-type GC (Table IV). In subgroup analysis based on tumor location, a significantly increased GC risk was observed in both cardia and non-cardia subgroups, but the effect was larger in the non-cardia GC (Table IV).

rs2976392 polymorphism and GC risk. Five studies were eligible for meta-analysis $(11,13,15,17-18)$, and all the included patients were Asians. The result of the meta-analysis indicated that rs2976392 A/G polymorphism was associated with increased risk of $\mathrm{GC}$, but the difference was not statistically significant (dominant model: OR, 1.41; 95\% CI, 0.98-2.04; Table IV). Notably, in subgroup analysis based on Lauren's classification, significant association was detected in diffuse and intestinal subgroups, and the association was stronger for the diffuse histological type (Table IV). However, in subgroup analysis based on tumor location, a significantly increased GC risk was observed in the non-cardia subgroup (dominant model, Table IV), but not in the cardia subgroup.

Association of PSCA polymorphisms with the survival of $G C$ patients. Only two included studies analyzed the association of PSCA rs2294008 genotypes with GC survival (20,21), and the results of the two studies were opposing. Therefore, we did not combine the data here. Wang et al (21) found no significant association between the genotypes and the survival of GC; however, by using stratified analysis according to Lauren's classification they found that the patients with rs2294008 CT/TT variant genotypes had a $25 \%$ significantly increased survival (HR, 0.75; 95\% CI, 0.59-0.96), compared to the CC homozygote among the diffuse-type GC. By contrast, Zeng et al (20) revealed that the TT genotype was associated with poor prognosis in GC patients (HR, 2.12; 95\% CI, 1.22-3.69). 
Publication bias. One funnel plot of the outcome of the rs2294008 polymorphism and GC risk in included studies demonstrated symmetry, indicating no serious publication bias (Fig. 2).

\section{Discussion}

Despite recent progress in the treatment of $\mathrm{GC}$, the prognosis of GC patients remains poor. To the best of our knowledge, stage is the best available clinical measure of tumor aggression and prognosis, but there are significant differences even within the same tumor stage (22). Therefore, discovery of new biomarkers and their application, in conjunction with traditional cancer diagnosis, staging and prognosis, may improve early diagnosis and reasonable care to a large extent. Previous studies have focused on the detection of genetic variants that are correlated with the development and progression of GC (23).

Certain Investigators have proposed that PSCA is involved in intracellular signaling, but much remains unknown regarding its physiological function and regulatory mechanism in normal and cancer cells. PSCA is up-regulated in prostate cancer and its expression is positively correlated with advanced clinical stage and metastasis in prostate cancer. Therefore, PSCA has been considered as a biomarker of diagnosis and prognosis, as well as a target of therapy for prostate cancer (8). By contrast, PSCA is down-regulated in GC and may have tumor-suppressing function in the gastric epithelium (10). Promising observations that genetic variations of PSCA (rs2976392 and rs2294008) are closely associated with the risk and survival of GC have opened up a new avenue of research regarding the pathological function of PSCA $(11,20)$. Here, we conducted a meta-analysis in order to validate the association between these PSCA polymorphisms and GC risk and survival. We found that $\mathrm{rs} 2294008 \mathrm{C} / \mathrm{T}$ (dominant model: OR, 1.44; 95\% CI, 1.16-1.79) and rs2976392 A/G (dominant model: OR, 1.41; 95\% CI, 0.98-2.04) polymorphisms were associated with increased risk of GC, although the association of rs2976392 was not statistically significant.

Two studies from different countries confirmed the significant association between PSCA polymorphisms and GC risk $(13,18)$. Notably, the frequency of risk T allele of rs2294008 and risk A allele of rs2976392 in populations of Japanese (0.617 and 0.616 , respectively) was more common than those in Chinese (0.256 and 0.244 , respectively), indicating the potential genetic heterogeneity among different populations. Therefore, we performed subgroup analysis according to ethnicity in this meta-analysis. We found that rs2294008 polymorphism was significantly associated with GC risk in Asians (dominant model: OR, 1.46; 95\% CI, 1.13-1.90) and Caucasians (recessive model: OR, 1.44; 95\% CI, 1.20-1.72). However, subgroup analysis was not conducted for rs 2976392 as all the included patients were Asians, but the result of metaanalysis indicated that the rs2976392 polymorphism was not significantly associated with GC risk in Asians (dominant model: OR, 1.41; 95\% CI, 0.98-2.04). This result differed from those of previous studies, which reported that the two SNPs were significantly associated with GC risk, and that the associations were weaker in Chinese than in Japanese patients $(11,13)$. The possible explanation for the difference may be the different ethnic population with genetic heterogeneity. More

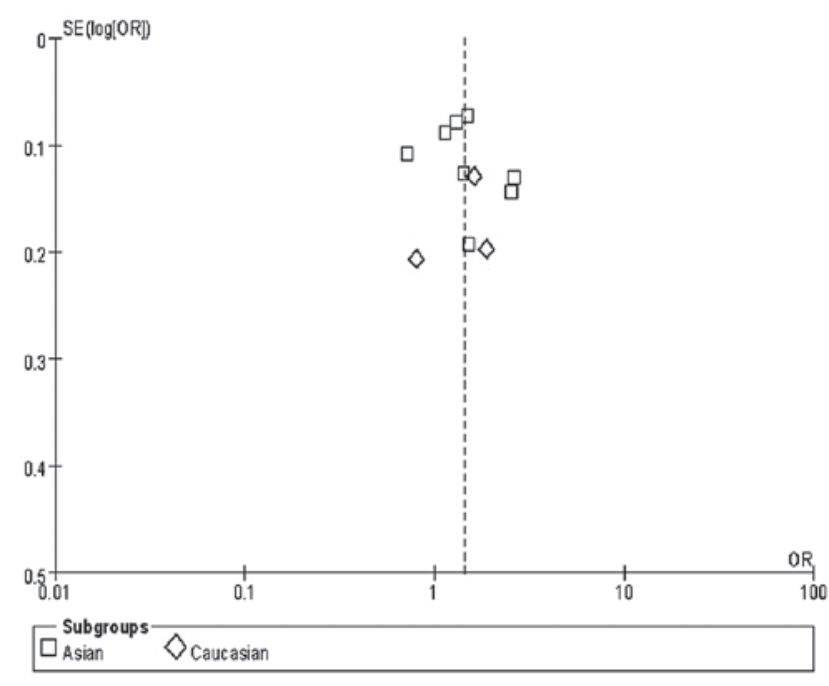

Figure 2. Funnel plot indicates no serious publication bias.

case-control studies with larger sample sizes, particularly studies evaluating rs2976392 in Caucasians, are required to further confirm these.

PSCA polymorphisms were found to be correlated with intestinal- and diffuse-type GC risk, and the effect was greater in diffuse-type GC $(11,18)$. Additionally, investigators proposed that the difference between intestinal- and diffuse-type GC with respect to genetic susceptibility is congruous with the hypothesis that two distinct pathways of gastric carcinogenesis exist: One arising in atrophic gastritis with or without intestinal metaplasia, which develops into intestinal-type GC at least initially, and the other originating from stem cells or precursors for gastric epithelial cells in the background of normal gastric mucosa, typically leading to diffuse-type cancer (24). In this meta-analysis, we found the rs2294008T allele was non-significantly associated with risk of diffuse-type (dominant model: OR, 1.42; 95\% CI, 0.89-2.27) or intestinal-type (dominant model: OR, 1.30; 95\% CI, 0.911.86) GC; however, rs2976392A allele was significantly correlated with both diffuse-type (dominant model: OR, 2.80; 95\% CI, 1.43-5.47) and intestinal-type (dominant model: OR, 1.46; 95\% CI, 1.27-1.69) GC. This result was not consistent with those of the majority of previous studies, but it was the same as the study by $\mathrm{Lu}$ et al (13). The possible reason for the difference was that we included patients with different characteristics, such as ethnicity, sample size, frequencies of allele and tumor location.

Studies have found that PSCA is mainly expressed in stomach nerve endocrine cells located in the stomach body and bottom gland (25), and this was validated by subsequent clinical trials, which showed that PSCA rs2294008 T allele was associated with significantly increased risk of non-cardia, no or decreased risk of cardia GC $(12,17,20)$. We found that the rs2294008 $\mathrm{T}$ allele was significantly associated with an increased risk of cardia and non-cardia GC, and the effect was larger in non-cardia (dominant model: OR, 1.45; 95\% CI, 1.26-1.66) than in cardia GC (dominant model: OR, 1.21; 95\% CI, 1.03-1.42). In addition, our meta-analysis revealed a positive association between rs2976392 and non- 
cardia GC but no association with cardia cancer (Table IV). These findings were compatible with those of the studies above-mentioned, suggesting that the germline variations in PSCA may be specific genetic markers for susceptibility to non-cardia GC development. A possible explanation is that cardia GC has different epidemiological characteristics, pathogenesis and clinical behaviors than non-cardia GC (26).

The majority of previous studies has focused on the association between PSCA polymorphisms and GC susceptibility, and few mention the prognostic value of PSCA polymorphisms to GC patients. In this review, we identified two studies examining the prognostic value of rs2294008 genotypes to GC patients, and the results were completely contrary $(20,21)$. Studies demonstrated that substitution of the $\mathrm{C}$ allele with the risk allele $\mathrm{T}$ reduced transcriptional activity of PSCA (11), and that decreased expression of PSCA may cause a decrease in cell adhesion molecules, which usually retain tumor cells at the primary site of carcinogenesis, thus increasing the chance of metastasis formation and decreasing the survival of GC patients $(27,28)$. This was consistent with the study by Zeng et al, who reported that the TT genotype was associated with the poor prognosis of GC patients (HR, 2.12; 95\% CI, 1.22-3.69) (20). By contrast, Wang et al observed that patients with rs2294008 CT/TT variant genotypes had a $25 \%$ significantly increased survival (HR, 0.75; 95\% CI, 0.59-0.96) (21). One potential reason for their result is that the reduced PSCA expression may increase sensitivity of GC cells to chemo-/radiotherapy, thus conferring improved survival; another reason may be due to different treatments in the two studies. However, the exact regulation mechanisms of PSCA expression and its biological function are largely unknown. Clinical trials analyzing the role of PSCA rs2294008 in GC patients receiving the same protocol of anticancer drugs are warranted to examine whether PSCA can serve as a potential prognostic marker.

Located in intron 2 of PSCA, the association of rs2976392 to GC susceptibility was evaluated by very few studies compared to rs2294008. Certain investigators found that the functional relevance of $\mathrm{rs} 2976392 \mathrm{G}>\mathrm{A}$ change was in strong linkage disequilibrium (LD) with the rs2294008C >T SNP $(11,13)$. In this meta-analysis, we found that rs2294008 and rs2976392 were associated with increased risk of GC, although the association of rs2976392 was not statistically significant (dominant model: OR, 1.41; 95\% CI, 0.98-2.04). This further supports the findings of the two studies above. However, the functional significance of the SNP rs2976392 remains unclear. It is also likely that rs2976392 is in high LD with other potentially functional or causal SNPs contributing to the development of GC. Therefore, the priority of research is to find the molecular mechanism by which the PSCA gene polymorphisms (especially rs2976392) are associated with GC.

In conclusion, our study showed that PSCA gene polymorphisms were associated with increased risk of GC, and were correlated with subtypes of clinicopathological features and the prognosis of GC patients. Therefore, our data indicated that PSCA may play a significant role in gastric carcinogenesis and prediction of prognosis of GC patients. Additional studies are required to evaluate the molecular mechanisms by which PSCA gene polymorphisms are associated with GC in popula- tions with diverse clinicopathological features, and to validate the prognostic value in a larger number of patients.

\section{References}

1. Parkin DM, Bray F, Ferlay J and Pisani P: Global cancer statistics, 2002. CA Cancer J Clin 55: 74-108, 2005.

2. Crew KD and Neugut AI: Epidemiology of gastric cancer. World J Gastroenterol 12: 354-362, 2006

3. Shikata K, Doi Y, Yonemoto K, Arima H, Ninomiya T, Kubo M, et al: Population-based prospective study of the combined influence of cigarette smoking and Helicobacter pylori infection on gastric cancer incidence: the Hisayama study. Am J Epidemiol 168: 1409-1415, 2008.

4. Machado JC, Figueiredo C, Canedo P, Pharoah P, Carvalho R, Nabais $\mathrm{S}$, et al: A proinflamatory genetic profile increases the risk for chronic atrophic gastritis and gastric carcinoma. Gastroenterology 125: 364-371, 2003.

5. Canedo P, Durães C, Pereira F, Regalo G, Lunet N, Barros H, et al: Tumor necrosis factor alpha extended haplotypes and risk of gastric carcinoma. Cancer Epidemiol Biomarkers Prev 17: 2416-2420, 2008.

6. Dong J, Dai J, Zhang M, Hu Z and Shen H: Potentially functional COX-2 $-1195 \mathrm{G}>\mathrm{A}$ polymorphism increases the risk of digestive system cancers: A meta-analysis. J Gastroenterol Hepatol 25: 1042-1050, 2010

7. Zhao D, Sun T, Zhang X, Guo Y, Yu D, Yang M, et al: Role of CD14 promoter polymorphisms in Helicobacter pylori infection-related gastric carcinoma. Clin Cancer Res 13: 2362-2368, 2007.

8. Reiter RE, Gu Z, Watabe T, Thomas G, Szigeti K, Davis E, et al: Prostate stem cell antigen: a cell surface marker overexpressed in prostate cancer. Proc Natl Acad Sci USA 95: 1735-1740, 1998

9. Saeki N, Gu J, Yoshida T and Wu X: Prostate stem cell antigen: a Jekyll and Hyde molecule? Clin Cancer Res 16: 3533-3538, 2010.

10. Bahrenberg G, Brauers A, Joost HG and Jakse G: Reduced expression of PSCA, a member of the LY- 6 family of cell surface antigens, in bladder, esophagus and stomach tumors. Biochem Biophys Res Commun 275: 783-788, 2000.

11. Sakamoto H, Yoshimura K, Saeki N, Katai H, Shimoda T, Matsuno Y, et al: Genetic variation in PSCA is associated with susceptibility to diffuse-type gastric cancer. Nat Genet 40: 730-740, 2008.

12. Lochhead P, Frank B, Hold GL, Rabkin CS, Ng MTH, Vaughan TL, et al: Genetic variation in the prostate stem cell antigen gene and upper gastrointestinal cancer in white individuals. Gastroenterology 140: 435-441, 2011.

13. Lu Y, Chen J, Ding Y, Jin G, Wu J, Huang H, et al: Genetic variation of PSCA gene is associated with the risk of both diffuseand intestinal-type gastric cancer in a Chinese population. Int $\mathbf{J}$ Cancer 127: 2183-2189, 2010.

14. The Nordic Cochrane Centre, The Cochrane Collaboration: Review Manager (RevMan) version 5.1. Copenhagen: The Nordic Cochrane Centre, The Cochrane Collaboration, 2011.

15. Ou J, Li K, Ren H, Bai H, Zeng D and Zhang C: Association and haplotype analysis of prostate stem cell antigen with gastric cancer in Tibetans. DNA Cell Biol 29: 319-323, 2010.

16. Song HR, Kim HN, Piao JM, Kweon SS, Choi JS, Bae WK, et al: Association of a common genetic variant in prostate stem-cell antigen with gastric cancer susceptibility in a Korean population. Mol Carcinogen 50: 871-875, 2011.

17. Wu C, Wang G, Yang M, Huang L, Yu D, Tan W, et al: Two genetic variants in prostate stem cell antigen and gastric cancer susceptibility in a Chinese population. Mol Carcinogen 48: 1131-1138, 2009.

18. Matsuo K, Tajima K, Suzuki T, Kawase T, Watanabe M, Shitara K, et al: Association of prostate stem cell antigen gene polymorphisms with the risk of stomach cancer in Japanese. Int J Cancer 125: 1961-1964, 2009.

19. Sala N, Muñoz X, Travier N, Agudo A, Duell EJ, Moreno V, et al: Prostate stem-cell antigen gene is associated with diffuse and intestinal gastric cancer in Caucasians: Results from the EPICEURGAST study. Int J Cancer 130: 2417-2427, 2011.

20. Zeng Z, Wu X, Chen F, Yu J, Xue L, Hao Y, et al: Polymorphisms in prostate stem cell antigen gene rs2294008 increase gastric cancer risk in Chinese. Mol Carcinogen 50: 353-358, 2011.

21. Wang M, Bai J, Tan Y, Wang S, Tian Y, Gong W, et al: Genetic variant in PSCA predicts survival of diffuse-type gastric cancer in a Chinese population. Int J Cancer 129: 1207-1213, 2011. 
22. Catalano V, Labianca R, Beretta GD, Gatta G, De Braud F and van Cutsem E: Gastric cancer. Crit Rev Oncol Hematol 71: 127-164, 2009.

23. Becker KF, Keller $\mathrm{G}$ and Hoefler $\mathrm{H}$ : The use of molecular biology in diagnosis and prognosis of gastric cancer. Surg Oncol 9: 5-11, 2000.

24. Smith MG, Hold GL, Tahara E and El-Omar EM: Cellular and molecular aspects of gastric cancer. World J Gastroenterol 12: 2979-2990, 2006.

25. Schoeman JH, De Vos V and Van Aswegen G: Distribution of endocrine cells in the gut of the impala. Ondersteport J Vet Res 65: 31-35, 1998.
26. Heidl G, Langhans P, Mellin W, Bunte H and Grundmann E: Adenocarcinoma of esophagus and cardia in comparison with gastric carcinoma. J Cancer Res Clin Oncol 120: 95-99, 1993.

27. Moore ML, Teitell MA, Kim Y, Watabe T, Reiter RE, Witte ON, et al: Deletion of PSCA increases metastasis of TRAMP-induced prostate tumors without altering primary tumor formation. Prostate 68: 139-151, 2008.

28. Raff AB, Gray A and Kast WM: Prostate stem cell antigen: a prospective therapeutic and diagnostic target. Cancer Lett 277: 126-132, 2009. 